\title{
CFD simulation of a stratified flow at the inlet of a compact plate heat exchanger
}

\author{
M. Ahmad, J. F. Fourmigue, P. Mercier \& G. Berthoud \\ Commissariat à l'Energie Atomique, Grenoble, France
}

\begin{abstract}
The work described in this paper considers 3D CFD (computational fluid dynamics) simulations of an adiabatic stratified liquid-vapor flow at the inlet of a compact plate heat exchanger using the commercial CFD code "FLUENT" and an in-house code "NEPTUNE 3D" developed by CEA and EDF. An experimental loop is built up that represents a compact plate heat exchanger in which the liquid and vapor flow rates in the different channels are measured and the flow inside the cylindrical distributor of diameter greater than that of the inlet tube can be visualized. The numerical predictions showed the good agreement with the experimental measurements. The interfacial shear stress was calculated in a steady stratified flow and compared with the computed shear stresses by the two codes.
\end{abstract}

Keywords: two-phase flow, maldistribution, CFD simulation, separated phase model, compact heat exchangers.

\section{Introduction}

Mal-distribution of two phase flow is the main cause of the deterioration of the thermal and hydraulic performances of heat exchangers and it is mainly affected by the flow pattern at the condensers or evaporators inlets (Ahmad et al [1]). The purpose of our study is to find a modeling tool capable of simulating some possible two phase flow patterns in compact heat exchangers. One of the most current flow patterns observed in the inlet tube of a heat exchanger is the stratified flow.

Few authors have tested the CFD simulation models to study the distribution in heat exchangers. Jones and Galliera [4] used FLUENT to simulate singlephase flow distribution in the manifold that performed well in calculating the 
larger scale features of branching and manifold flows. Lalot et al [5] used the computer code STAR-CD to study the flow maldistribution in an electric heater. Zhang and Li [7] used FLUENT to predict the flow distribution in plate-fin heat exchangers. Fei [2] used the mixture model implemented in FLUENT to simulate a two-phase flow distribution in the header.

CFD simulation technique can provide the flexibility to construct computational models that can be easily adapted to a wide variety of physical conditions without constructing a large scale prototype or expensive test rigs. In our study, a stratified liquid-vapour flow in a compact heat exchanger is simulated using the commercial CFD code "FLUENT" and an in-house code "NEPTUNE CFD" developed by CEA and EDF. The interfacial shear behavior has a great influence in this kind of configurations. Therefore, the simulations were first carried out using the single-fluid approach in combination with the VOF model implemented in FLUENT and then the two phase approach of NEPTUNE CFD with the adaptation of a dispersed flow shear force model for a separated-phases flow. The numerical results of the two codes are compared with the experimental data.

\section{Study of the flow distribution}

\subsection{Experimental apparatus}

A distribution experimental loop representing a compact plate heat exchanger was built up. The test section consists of a horizontal manifold and eight parallel downward branches (Figure 1).

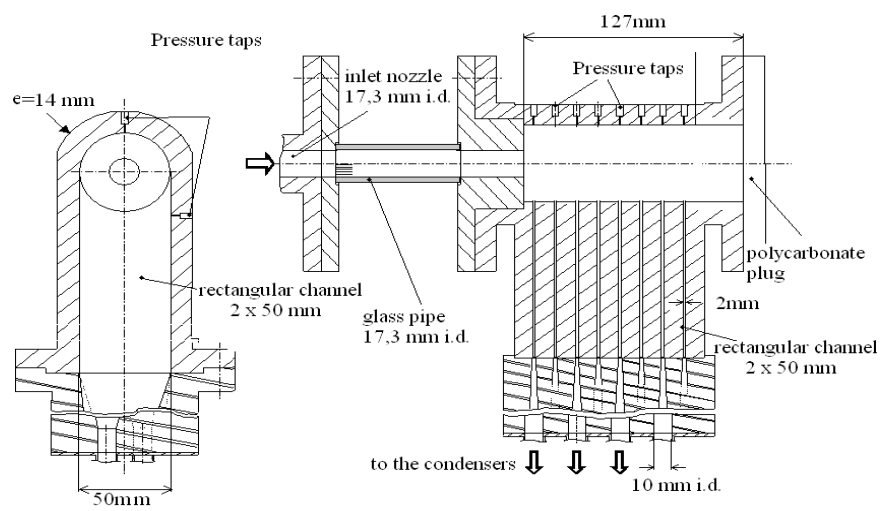

Figure 1: $\quad$ Test section.

The manifold is $127 \mathrm{~mm}$ long and its diameter is $50 \mathrm{~mm}$. It is horizontally supplied by a $17.3 \mathrm{~mm}$ in diameter and $100 \mathrm{~mm}$ long glass pipe to visualize the two phase flow at the header inlet with a $1,500 \mathrm{~mm}$ tube made of stainless steel of the same diameter. The end of the manifold is closed by a transparent 
polycarbonate plug. Each branch is $2 \times 50 \mathrm{~mm}$ rectangular. The channels are regularly $10 \mathrm{~mm}$ spaced along the manifold. Figure 2 shows an isometric view of the test section.

\subsection{CFD models}

In the two codes, the conservation equations of mass and momentum are solved using the finite volume method. The used mesh involved 250,000 cells (figure 3) making a compromise between the accuracy in representing the physical phenomena needing refined grid and the cpu-time. An average necessary cputime for each treated case varies from ten days with FLUENT to three weeks with NEPTUNE 3D.

In FLUENT, an automatic meshing allows the grid refinement at the interface level depending on the gradient of the void fraction and thus can decrease the numerical diffusion in the value of the void fraction in this zone. The turbulence was modelled using the RNG-based $k-\varepsilon$ turbulence model in FLUENT [3] and $k-\varepsilon$ EDF model [6] in NEPTUNE.

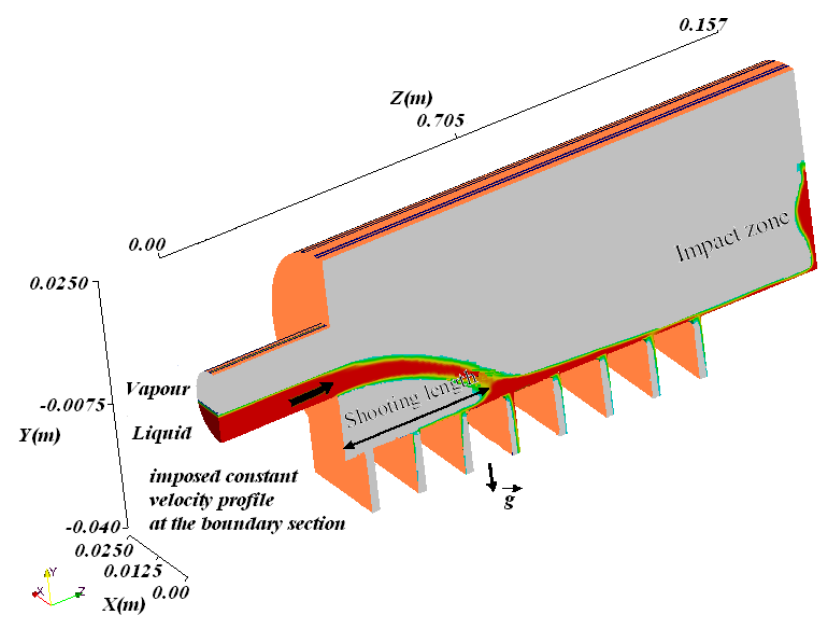

Figure 2: An isometric cross section of the header.

\subsubsection{FLUENT-VOF}

The VOF model implemented in FLUENT is a one-fluid model. It relies on the fact that the two phases are not interpenetrating. The volume fraction of each phase is calculated in each computational cell. The variables and properties of each cell are either representative of one phase or representative of the mixture of the two phases, depending on the volume fraction value. The tracking of the interface between the two phases is accomplished by the solution of the continuity equation for the volume fraction of one of the phases. 


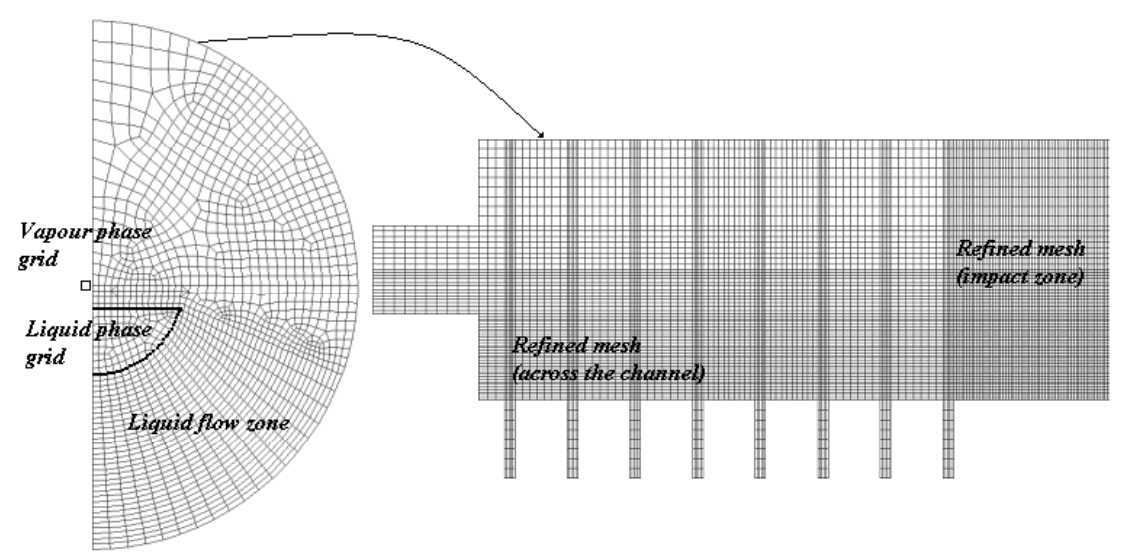

Figure 3: Over view of the mesh.

$$
\frac{\partial \alpha_{q}}{\partial t}+\vec{v} \cdot \nabla \alpha_{q}=0
$$

$\alpha_{q}$ is the volume fraction of phase $q$ and $\vec{v}$ is the vector velocity. A single momentum conservation equation is solved through out the domain and the resulting velocity field is shared among the two phases. The momentum equation, shown below, is dependent on the volume fraction of all phases through the properties $\rho$ and $\mu$.

$$
\frac{\partial}{\partial t}(\rho \vec{v})+\nabla \cdot(\rho \vec{v} \vec{v})=-\nabla P+\nabla \cdot\left[\mu\left(\nabla \vec{v}+\nabla \vec{v}^{T}\right)\right]+\rho \vec{g}+\vec{F}
$$

where $P$ is the pressure, $\rho=\sum \alpha_{q} \rho_{q}$ is the average mass density, $\mu=\sum \alpha_{q} \mu_{q}$ is the average dynamic viscosity and $\vec{F}$ is the surface tension force.

\subsubsection{NEPTUNE 3D-two fluid model}

The balance equations of the two fluid model where the Navier-Stokes equation apply for each phase can be written:

- Two mass balance equations:

$$
\frac{\partial}{\partial t}\left(\alpha_{q} \rho_{q}\right)+\nabla \cdot\left(\alpha_{q} \rho_{q} \vec{v}_{q}\right)=0
$$

- Two momentum balance equations:

$$
\alpha_{q} \rho_{q}\left[\frac{\partial \vec{v}_{q}}{\partial t}+\left(\vec{v}_{q} \cdot \nabla \vec{v}_{q}\right)\right]=\nabla \cdot\left[\alpha\left(\overline{\bar{\tau}}_{q}+\overline{\bar{\tau}}_{q}^{\mathrm{Re}}\right)\right]-\alpha_{q} \vec{\nabla} P+\alpha_{q} \rho_{q} \vec{g}+\vec{M}_{q i}
$$


$\overline{\bar{\tau}}_{q}$ is the shear tensor and $\vec{M}_{q i}$ is the interfacial momentum transfer term that accounts for mass transfer, drag force, added mass, lift,... In our treated cases, the drag force is the only considered interfacial force, thus $M_{q i}=\tau_{q i} a_{i}$, where $\tau_{q i}$ is the interfacial friction per unit area and $a_{i}$ is the volumetric interfacial area.

- $\quad$ Drag force

The interfacial drag force is calculated with the adaptation of a dispersed flow shear force model to compute a separated-phases flow. The interfacial drag transfer term between phases $p$ and $q$ has the following form:

$$
I_{(p \rightarrow q)}=-\left(\alpha_{p} \alpha_{q}\right) F_{D} V_{r}^{p q}
$$

$F_{D}$, drag coefficient between phases $p$ and $q . V_{r}^{p q}$, relative velocity between phases $p$ and $q, \alpha$ is the volumetric fraction. The drag coefficient is written in terms of the particle drag-relaxation time $\tau_{p}$. In the following notation, $p$ represents the dispersed phase and $q$ represents the continuous phase:

$$
F_{D}=\frac{\rho_{p}}{\alpha_{q}} \frac{1}{\tau_{p}}, \frac{1}{\tau_{p}}=\frac{\rho_{q}}{\rho_{p}} \frac{3}{4} \frac{C_{D}}{d_{p}}<\left|V_{r}^{p q}\right|>_{p}, C_{D}=\frac{24}{\operatorname{Re}_{p}}\left[1+0.15 \operatorname{Re}_{p}^{0.687}\right]
$$

The model considers either dispersed gas (vapour $V$ ) bubbles in a continuous liquid $(L)$ flow, or dispersed liquid droplets in a continuous gas (vapour) flow with regard to the volumetric fraction.

$$
\begin{gathered}
\text { - } \quad \text { Bubbly flow }\left(\alpha_{V}<0.3\right) \quad F_{D}^{\text {bubble }}=\frac{\rho_{V}}{\alpha_{L}} \frac{1}{\tau_{V}}, \\
\text { - } \quad \text { Misty flow }\left(\alpha_{V}>0.7\right) \quad F_{D}^{\text {droplet }}=\frac{\rho_{L}}{\alpha_{V}} \frac{1}{\tau_{L}} \\
-\quad \text { Mixing }\left(0.3<\alpha_{V}<0.7\right): \\
F_{D}=\left(\frac{0.7-\alpha_{V}}{0.7-0.3}\right) F_{D}^{\text {bubble }}(0.3)+\left(\frac{\alpha_{V}-0.3}{0.7-0.3}\right) F_{D}^{\text {droplet }}(0.7)
\end{gathered}
$$

we can note that for this closure, two characteristic diameters have to be defined. In our case of liquid-vapour separated flow (stratified), the volumetric fraction $\left(\alpha_{p}\right)$ tends to zero (or a residual value) in the two single phase zones, and included between 0.3 and 0.7 (arbitrary) in the interface zone.

\subsection{Results and discussion}

The validation of the computed results is built up basically on the comparison between the numerical predictions of the two-phase distribution in the different channels and the experimental data of the treated cases. In the presentation of the 
results of the two-phase distribution measurements, the non-dimensional liquid (resp. vapour) flow rate in channel $i$ is the ratio of the liquid (resp. vapour) flow rate measured in the channel over the mean liquid (resp. vapour) flow rate:

$$
\dot{M}_{\mathrm{k} i}^{*}=\frac{\dot{M}_{\mathrm{k} i}}{\sum_{j=1}^{8} \dot{M}_{\mathrm{k} j} / 8}
$$

where $\mathrm{k}=\ell$ (liquid) or $\mathrm{k}=v$ (vapour). Two different cases are treated corresponding to a stratified flow at the header inlet. In the first case, a total inlet flow rate of $86 \mathrm{~kg} / \mathrm{hr}$ (average superficial mass velocity is $100 \mathrm{~kg} / \mathrm{m}^{2} \mathrm{~s}$ ) is imposed with a mass quality of $20 \%$. In the second case, we increased the flow rate to $129 \mathrm{~kg} / \mathrm{hr}$ (average superficial mass velocity is $150 \mathrm{~kg} / \mathrm{m}^{2} \mathrm{~s}$ ) with the same mass quality and void fraction. A homogeneous mean velocity profile $\left(\bar{V}_{K}\right)$ is fixed for each phase in the boundary section.

$$
\bar{V}_{L}=\frac{G_{T}(1-x)}{\rho_{L}(1-\alpha)}, \bar{V}_{V}=\frac{G_{T} x}{\rho_{V} \alpha}
$$

$G_{T}\left(\mathrm{~kg} / \mathrm{m}^{2} . \mathrm{s}\right)$ is the average inlet superficial mass velocity, $\rho_{L}$ and $\rho_{V}$ are respectively the mass densities of liquid and vapour, $x$ is the mass quality and $\alpha$ is the void fraction calculated using the model of Lockhart-Martinelli for a steady state two-phase flow. Being in an instationary flow, comparisons requested averaged time values for computed and experimental date.
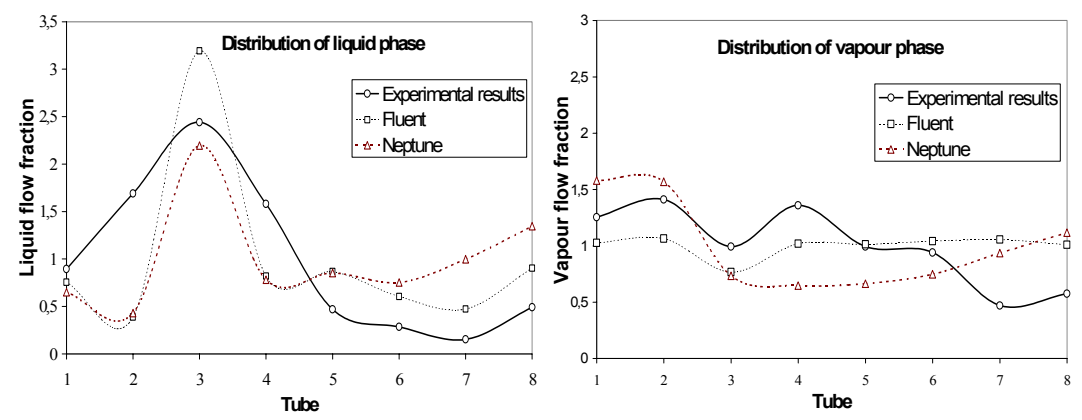

Figure 4: Comparison between numerical predictions and experiment (case1: $\mathrm{G}=100 \mathrm{~kg} / \mathrm{m}^{2} \mathrm{~s}, \mathrm{x}=20 \%$ ).

\subsubsection{Case 1}

Figure 4 shows that both numerical predictions and experimental data have a similar distribution profile for the liquid phase. The liquid flow rates in the channels 2 and 4 are under-predicted by the two solvers. In last four channels, the computed liquid flow rates are higher than the measured values. FLUENT- 
VOF solver seems to perform better than the code NEPTUNE in the last four channels, whereas Neptune gives better results in channel 3.

The prediction of the vapour distribution in figure 4 shows a maximum discrepancy of $60 \%$ of the mean vapour flow with FLUENT in channel 7 and $70 \%$ of the mean vapour flow rate with NEPTUNE in channel 4 when compared to experimental measurements.

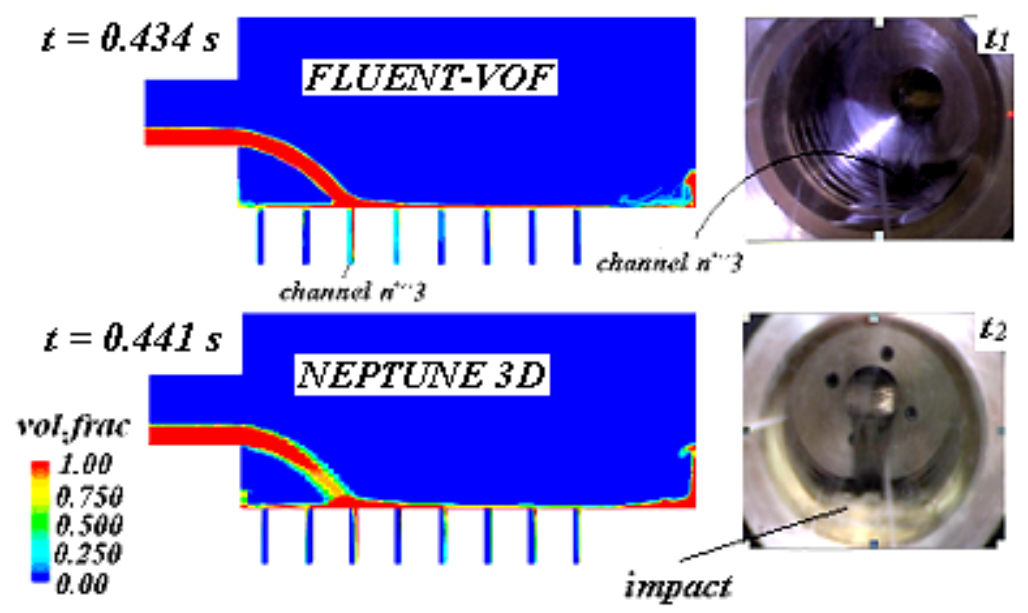

Figure 5: Contours of computed volume fractions and visualizations (case 1: $\mathrm{G}=100 \mathrm{~kg} / \mathrm{m}^{2} \mathrm{~s}, \mathrm{x}=20 \%$ ).

The liquid vapour interface traced by the two codes as illustrated in figure 5 shows almost the same shooting length after the inlet section enlargement. Figure 5 also shows in the right side, the visualization of the instationary flow at two instants $t_{1}$ and $t_{2}$. At $t_{1}$, it shows the shooting point at channel 3 level as predicted by the two codes and at $t_{2}$, it shows the liquid jump at the header cap. The interfacial shear forces did not seem to have a significant influence in this part of the flow compared to the inertia force. However, NEPTUNE slightly over estimated shear forces (see section 3 ) which resulted in a slightly higher liquid jump after the impact than that was shown by visualizations (instant $t_{2}$ ).

\subsubsection{Case 2}

In the second case, the total inlet flow rate was increased, mass quality was fixed and thus the void fraction was the same but with higher liquid momentum. The two codes also give a similar distribution profile for the liquid phase (figure 6). The uncertainty of FLUENT-VOF code prediction is less than $25 \%$ of the mean liquid flow rate except for channels 3 and 4 where it reaches $60 \%$. The prediction of Neptune code reaches $200 \%$ of the mean liquid flow rate in the last channel. 
The vapour distribution is better predicted by FLUENT code. The error in the prediction is less than or equal to $25 \%$ of the mean vapour flow rate except for the last channel where the difference is estimated to $45 \%$.
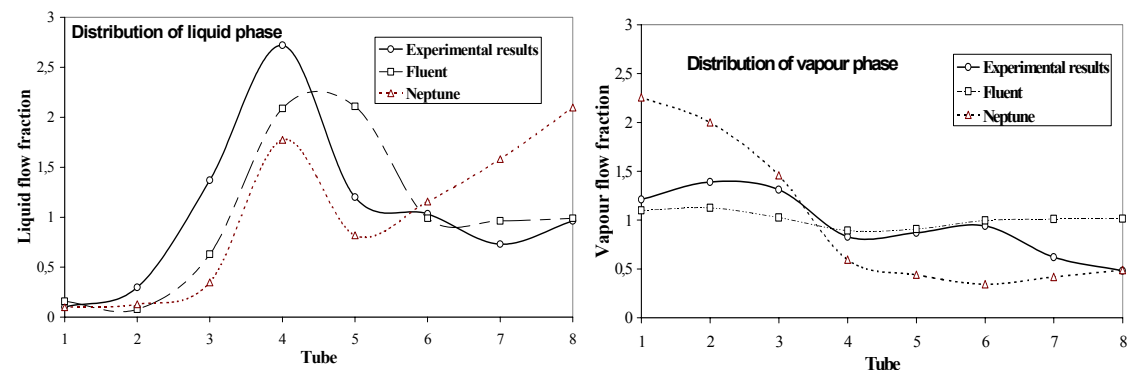

Figure 6: Comparison between numerical predictions and experiment (case 2: $\mathrm{G}=150 \mathrm{~kg} / \mathrm{m}^{2} \mathrm{~s}, \mathrm{x}=20 \%$ ).

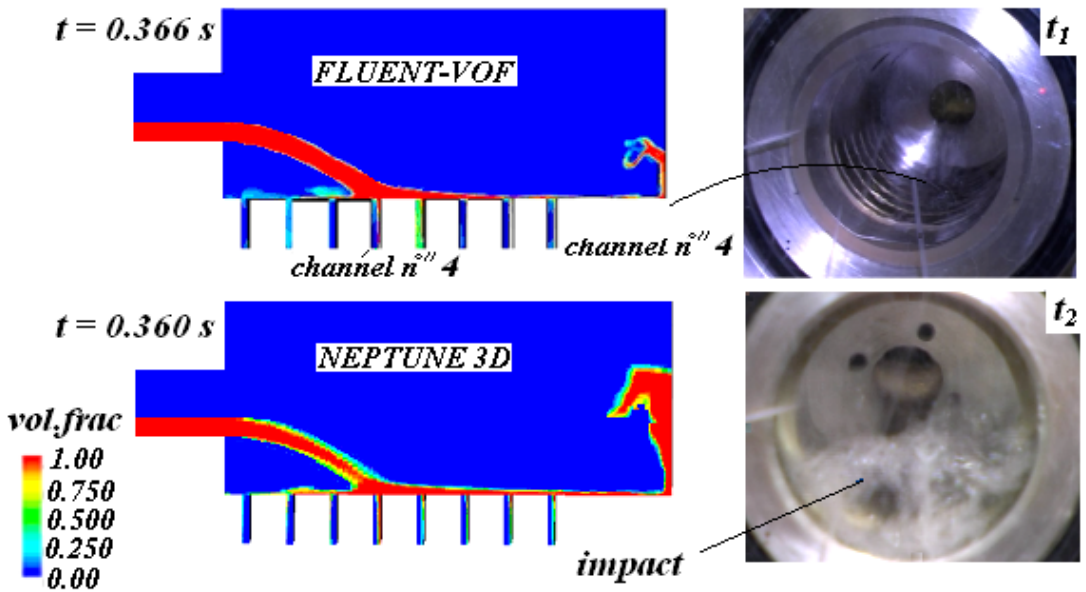

Figure 7: Contours of volume fractions and visualizations (case2: $G=150$ $\mathrm{kg} / \mathrm{m}^{2} \mathrm{~s}, \mathrm{x}=20 \%$ ).

The computed mass and velocity of the liquid after channel 8 are much higher in NEPTUNE code which is illustrated by a much higher liquid jump after the impact when compared with visualizations (figure 7). This discrepancy might be explained by the different grid resolution used in the two codes and the important shear force calculated in NEPTUNE. 


\section{Calculation of the average interfacial shear in a steady stratified flow}

In this section, a model was carried out to estimate the average of the interfacial shear stress in a steady stratified flow between two parallel plates. The velocity profiles were defined for the two phases using the one-seventh law for a turbulent flow. The vapour velocity is defined in two zones, the first zone is between the wall and the point of maximum velocity value (zone of thickness $b$ ) and the second zone is between the liquid-vapour interface and that point of maximum velocity value (zone of thickness $a$ ) as shown in figure 8 .
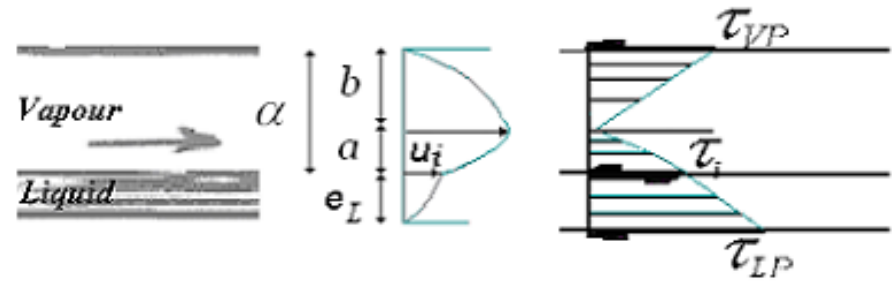

Figure 8: Liquid-vapour flow in a steady state in a parallel plate channel.

The equilibrium between the pressure gradient motive force and the boundary shear forces can be applied on each phase as well as on both phases. The pressure drop in the liquid and in the vapour phase is the same as it is in the two phases, so we can write:

$$
\frac{\Delta p}{\Delta l}=\frac{\tau_{L p}-\tau_{i}}{e_{L}}=\frac{\tau_{V p}+\tau_{L p}}{D}
$$

$\tau_{i}$ is the average value of the interfacial shear stress, between liquid and vapour phases in a steady state flow. $\tau_{L p}$ and $\tau_{V p}$ are respectively the averaged shear stresses between the liquid and the vapour with wall. $\mathrm{D}$ is the thickness of the channel and $e_{L}$ is the thickness of the liquid phase, and thus we can write:

$$
e_{L}=D(1-\alpha)
$$

$\alpha$ being the void fraction that can be calculated using the model of LockhartMartinelli:

$$
a+b=\alpha D
$$

To describe the velocity profiles of both phases, the one seventh-law of a turbulent flow was used. For the vapour, this law was applied in the two zones that are joined at the point of the same maximum vapour velocity.

$$
U_{V 1}=8.74 v_{V} \frac{-1}{7}\left(\frac{\tau_{V p}}{\rho_{V}}\right)^{\frac{4}{7}} y_{2}^{\frac{1}{7}}, \quad 0<y_{2}<b
$$




$$
U_{V 2}=8.74 v_{V} \frac{-1}{7}\left(\frac{\tau_{i}}{\rho_{V}}\right)^{\frac{4}{7}} y_{1}^{\frac{1}{7}}+u_{i}, \quad 0<y_{1}<a
$$

At the point of maximum vapour velocity, equality between equations (15) and (16) is attained, at $y_{1}=a$ and $y_{2}=b$, we have:

$$
\begin{gathered}
U_{V 1(\max )}=U_{V 2(\max )} \Rightarrow \\
8.74 v_{V} \frac{-1}{7}\left(\frac{\tau_{V p}}{\rho_{V}}\right)^{\frac{4}{7}} b^{\frac{1}{7}}=8.74 v_{V} \frac{-1}{7}\left(\frac{\tau_{i}}{\rho_{V}}\right)^{\frac{4}{7}} a^{\frac{1}{7}}+u_{i}
\end{gathered}
$$

The vapour flow rate can be expressed as a function of the mean vapour velocity as follows:

$$
Q_{V}=\bar{V}_{V}\left(D-e_{L}\right)
$$

By integration and summation of equations (15) and (16), we can deduce the vapour flow rate:

$$
Q_{V}=\bar{V}_{V} \cdot\left(D-e_{L}\right)=7.6125 v_{V}^{\frac{-1}{7}} \rho_{V}^{\frac{-4}{7}}\left[a^{\frac{8}{7}} \tau_{i}^{\frac{4}{7}}+b^{\frac{8}{7}} \tau_{V p}^{\frac{4}{7}}\right]+u_{i} a
$$

Similarly, using the one-seventh law we can describe the liquid velocity profile:

$$
U_{L}=8.74 v_{L} \frac{-1}{7}\left(\frac{\tau_{L p}}{\rho_{L}}\right)^{\frac{4}{7}} y^{\frac{1}{7}}, \quad 0<y<e_{L}
$$

The interfacial velocity can be then deduced from equation (20):

$$
u_{i}=8.74 v_{L}^{\frac{-1}{7}}\left(\frac{\tau_{L p}}{\rho_{L}}\right)^{\frac{4}{7}} e_{L}^{\frac{1}{7}}
$$

As for vapour phase, by integration of equation (20), we can get the liquid flow rate function of the mean liquid velocity:

$$
Q_{L}=\bar{V}_{L} \cdot e_{L}=7.6475 v_{L}^{-\frac{1}{7}}\left(\frac{\tau_{L p}}{\rho_{L}}\right)^{\frac{4}{7}} e_{L}^{\frac{8}{7}}
$$

Knowing the hydraulic diameter of the plate, the mass quality and inlet flow rate, the resolution of the system of equations gives the average steady state value of the interfacial shear force.

Figure 9 represents the comparison between the calculated value of the shear force in a channel having the same hydraulic diameter $(17.3 \mathrm{~mm})$ and initial boundary conditions as in case 1 (section 2.3) and the computed shear forces. The shear force value of FLUENT-VOF solver in the interface cell is computed using an average dynamic viscosity value between the two phases. Increasing the bubble characteristic diameter decreases the NEPTUNE computed shear force value and decreases the error of the prediction. 


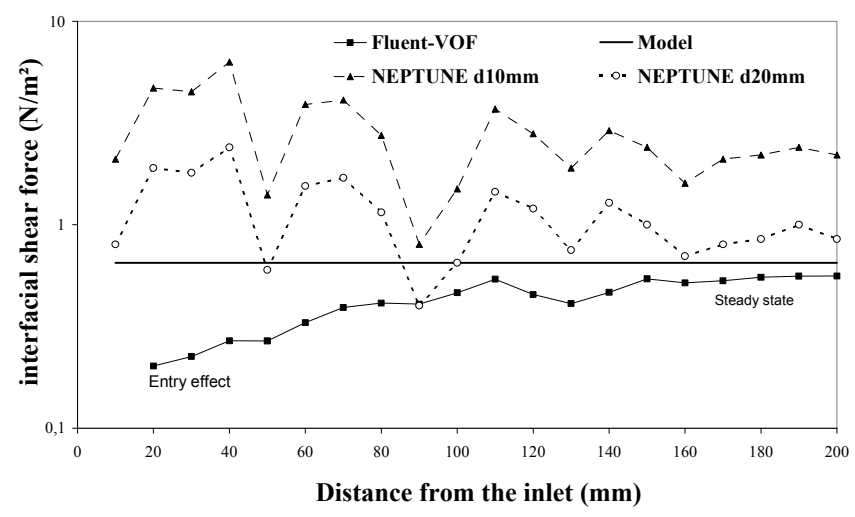

Figure 9: Comparison between model and computed values of shear forces.

\section{Conclusion}

A stratified liquid-vapour flow at the inlet of a compact heat exchanger was simulated using "FLUENT" and "NEPTUNE" CFD codes and experimental measurements have been carried out. The profile distributions of both phases in all channels were rather well predicted by the two codes. However FLUENTVOF solver seems to perform better than NEPTUNE in the case of high liquid inlet momentum (case 2). This error in the prediction by NEPTUNE might be due to the insufficient grid resolution and the over-estimation of shear force of dispersed flow model.

\section{References}

[1] Ahmad, M., Mercier, P. and Berthoud, G., Experimental study of twophase distribution in a compact plate heat exchanger, Inter Conf, Heat SET, GRETh/CEA, Chambery 2007.

[2] Fei, P., Adiabatic developing two-phase refrigerant flow in manifolds of heat exchangers, thesis of University if Illinois at Urbana Champaign, 2003.

[3] FLUENT 6, Dec. 2001 User Guide. Fluent Inc.

[4] Jones, G. F. and Galliera, J. M., Isothermal Flow Distribution in Coupled Manifolds: Comparison of Results from CFD and an Integral Model, Proc. ASME Int. Cong. and Exhib., Anaheim, CA, 1998.

[5] Lalot, S., Florent, P. and Lang, S. K., Flow maldistribution in heat exchangers. Appl. Thermal Eng. 26, pp. 847-863, 1999.

[6] Lavieville, J. Bouker, M. and al., NEPTUNE CFD V1.0, Theory Manual, EDF R\&D, Chatou, 2006.

[7] Zhang, Z. and Li, Y. Z., CFD simulation on inlet configuration of plate-fin heat exchangers, School of Energy and Power Engineering, Xi'an 710049, China, 2003. 